\title{
Primeness and primitivity conditions for twisted group $C^{*}$-algebras
}

\author{
Tron Ånen Omland *
}

October 12, 2018

\begin{abstract}
For a multiplier (2-cocycle) $\sigma$ on a discrete group $G$ we give conditions for which the twisted group $C^{*}$-algebra associated with the pair $(G, \sigma)$ is prime or primitive. We also discuss how these conditions behave on direct products and free products of groups.
\end{abstract}

\section{Introduction}

In this paper, $G$ will always denote a discrete group with identity $e$. The full group $C^{*}$-algebra associated with $G, C^{*}(G)$ is simple only if $G$ is trivial, but other aspects of its ideal structure are of interest. Recall that a $C^{*}$-algebra is called primitive if it has a faithful irreducible representation and prime if nonzero ideals have nonzero intersection. Primeness of a $C^{*}$-algebra is in general a weaker property than primitivity. However, according to a result of Dixmier 9, the two notions coincide for separable $C^{*}$-algebras.

Furthermore, recall what the icc property means for $G$ - that every nontrivial conjugacy class is infinite, and its importance comes to light in the following theorem.

Theorem A. The following are equivalent:

(i) G has the icc property.

(ii) The group von Neumann algebra $W^{*}(G)$ is a factor.

(iii) The reduced group $C^{*}$-algebra $C_{r}^{*}(G)$ is prime.

The equivalence (i) $\Leftrightarrow$ (ii) is a well known result of Murray and von Neumann [19], while (i) $\Leftrightarrow$ (iii) is proved by Murphy [18]. Murphy also shows that the icc property is a necessary condition for primeness of $C^{*}(G)$. Therefore, for the class of discrete groups, primeness and, in the countable case, primitivity, may be regarded as $C^{*}$-algebraic analogs of factors. The theorem gives as a corollary that if $G$ is countable and amenable, then primitivity of $C^{*}(G)$ is equivalent with the icc property of $G$. Moreover, since amenability of $G$ implies injectivity

\footnotetext{
2010 Mathematics Subject Classification. Primary 46L05; Secondary 22D25, 20C25

Key words and phrases. twisted group $C^{*}$-algebra, primitivity, primeness, projective unitary representation, multiplier, amenable group.

* Partially supported by the Research Council of Norway
} 
of $W^{*}(G)$, this is also equivalent to $W^{*}(G)$ being the hyperfinite $\mathrm{II}_{1}$ factor if $G$ is nontrivial, according to Connes [8].

In the present paper, Theorem $\mathrm{A}$ will be adapted to a twisted setting where pairs $(G, \sigma)$ consisting of a group $G$ and a multiplier $\sigma$ on $G$ are considered. We will show that the reduced twisted group $C^{*}$-algebra $C_{r}^{*}(G, \sigma)$ is prime if and only if every nontrivial $\sigma$-regular conjugacy class of $G$ is infinite, and say that the pair $(G, \sigma)$ satisfies condition $K$ if it possesses this property. It was first introduced by Kleppner [13, who proves that this property is equivalent to the fact that the twisted group von Neumann algebra $W^{*}(G, \sigma)$ is a factor. The main part of our proof is to show that $(G, \sigma)$ satisfies condition $\mathrm{K}$ if and only if $C_{r}^{*}(G, \sigma)$ has trivial center, and this argument is, of course, inspired by the mentioned works of Kleppner and Murphy. As a corollary, we get that primeness of the full twisted group $C^{*}$-algebra $C^{*}(G, \sigma)$ implies condition $\mathrm{K}$ on $(G, \sigma)$. The converse is not true in general, but at least holds if $G$ is amenable, as the full and reduced twisted group $C^{*}$-algebras then are isomorphic. Thus, if $G$ is countable and amenable, condition $\mathrm{K}$ on $(G, \sigma)$ is equivalent to primitivity of $C^{*}(G, \sigma)$ by applying Dixmier's result. This fact is also explained by Packer 21] with a different approach. On the other hand, no examples of nonprimitive, but prime twisted group $C^{*}$-algebras are known, so it is not clear whether we need the countability assumption on $G$.

In the last two sections we will investigate primeness and primitivity of the twisted group $C^{*}$-algebras of $(G, \sigma)$ when $G=G_{1} \times G_{2}$ and when $G=G_{1} * G_{2}$. The free product case turns out to be easier to handle in general, since the corresponding $C^{*}$-algebra always decomposes into a free product of the two components. For direct products, however, the multiplier $\sigma$ on $G$ can have a 'cross-term' which makes a $C^{*}$-algebra decomposition into tensor products impossible.

A significant part of this work, especially Section 2, was accomplished when I was a student at University of Oslo, and is also included in my master's thesis. I am indebted to Erik Bédos for his advice, both on the thesis and on the completion of this paper.

I would also like to thank the referee for several useful comments and suggestions.

\section{Twisted group $C^{*}$-algebras}

Let $G$ be a group and $\mathcal{H}$ a nontrivial Hilbert space. The projective unitary group $P U(\mathcal{H})$ is the quotient of the unitary group $U(\mathcal{H})$ by the scalar multiples of the identity, that is,

$$
P U(\mathcal{H})=U(\mathcal{H}) / \mathbb{T} 1_{\mathcal{H}} .
$$

A projective unitary representation $G$ is a homomorphism $G \rightarrow P U(\mathcal{H})$. Every lift of a projective representation to a map $U: G \rightarrow U(\mathcal{H})$ must satisfy

$$
U(a) U(b)=\sigma(a, b) U(a b)
$$

for all $a, b \in G$ and some function $\sigma: G \times G \rightarrow \mathbb{T}$. From the associativity of $U$ and by requiring that $U(e)=1_{\mathcal{H}}$, the identities

$$
\begin{gathered}
\sigma(a, b) \sigma(a b, c)=\sigma(a, b c) \sigma(b, c) \\
\sigma(a, e)=\sigma(e, a)=1
\end{gathered}
$$


must hold for all elements $a, b, c \in G$.

Definition. Any function $\sigma: G \times G \rightarrow \mathbb{T}$ satisfying (2) is called a multiplier on $G$, and any map $U: G \rightarrow U(\mathcal{H})$ satisfying (1D) is called a $\sigma$-projective unitary representation of $G$ on $\mathcal{H}$.

The lift of a homomorphism $G \rightarrow P U(\mathcal{H})$ up to $U$ is not unique, but any other lift is of the form $\beta U$ for some function $\beta: G \rightarrow \mathbb{T}$. Therefore, two multipliers $\sigma$ and $\tau$ are said to be similar if

$$
\tau(a, b)=\beta(a) \beta(b) \overline{\beta(a b)} \sigma(a, b)
$$

for all $a, b \in G$ and some $\beta: G \rightarrow \mathbb{T}$. Note that we must have $\beta(e)=1$ for this to be possible. We say that $\sigma$ is trivial if it is similar to 1 and call $\sigma$ normalized if $\sigma\left(a, a^{-1}\right)=1$ for all $a \in G$.

Moreover, the set of similarity classes of multipliers on $G$ is an abelian group under pointwise multiplication. This group is the Schur multiplier of $G$ and will henceforth be denoted by $\mathcal{M}(G)$. Also, we remark that multipliers are often called 2-cocycles on $G$ with values in $\mathbb{T}$, and that the Schur multiplier of $G$ coincides with the second cohomology group $H^{2}(G, \mathbb{T})$.

Let $\sigma$ be a multiplier on $G$. We will briefly explain how the operator algebras associated with the pair $(G, \sigma)$ are constructed and refer to Zeller-Meier 24 for further details. First, the Banach *-algebra $\ell^{1}(G, \sigma)$ is defined as the set $\ell^{1}(G)$ together with twisted convolution and involution given by

$$
\begin{aligned}
\left(f *_{\sigma} g\right)(a) & =\sum_{b \in G} f(b) \sigma\left(b, b^{-1} a\right) g\left(b^{-1} a\right) \\
f^{*}(a) & =\overline{\sigma\left(a, a^{-1}\right) f\left(a^{-1}\right)}
\end{aligned}
$$

for elements $f, g$ in $\ell^{1}(G)$, and is equipped with the usual $\|\cdot\|_{1}$-norm.

Definition. The full twisted group $C^{*}$-algebra $C^{*}(G, \sigma)$ is the universal enveloping algebra of $\ell^{1}(G, \sigma)$. Moreover, the canonical injection of $G$ into $C^{*}(G, \sigma)$ will be denoted by $i_{(G, \sigma)}$ or just $i_{G}$ if no confusion arise.

For $a$ in $G$, let $\delta_{a}$ be the function on $G$ defined by

$$
\delta_{a}(b)= \begin{cases}1 & \text { if } b=a, \\ 0 & \text { if } b \neq a .\end{cases}
$$

Then the set $\left\{\delta_{a}\right\}_{a \in G}$ is an orthonormal basis for $\ell^{2}(G)$ and generates $\ell^{1}(G, \sigma)$, so that for all $a$ in $G, i_{(G, \sigma)}(a)$ is the image of $\delta_{a}$ in $C^{*}(G, \sigma)$. The left regular $\sigma$-projective unitary representation $\lambda_{\sigma}$ of $G$ on $B\left(\ell^{2}(G)\right)$ is given by

$$
\left(\lambda_{\sigma}(a) \xi\right)(b)=\left(\delta_{a} *_{\sigma} \xi\right)(b)=\sigma\left(a, a^{-1} b\right) \xi\left(a^{-1} b\right) .
$$

Note in particular that

$$
\lambda_{\sigma}(a) \delta_{b}=\delta_{a} *_{\sigma} \delta_{b}=\sigma(a, b) \delta_{a b}
$$

for all $a, b \in G$. Moreover, the integrated form of $\lambda_{\sigma}$ on $\ell^{1}(G, \sigma)$ is defined by

$$
\lambda_{\sigma}(f)=\sum_{a \in G} f(a) \lambda_{\sigma}(a) .
$$


Definition. The reduced twisted group $C^{*}$-algebra and the twisted group von Neumann algebra of $(G, \sigma), C_{r}^{*}(G, \sigma)$ and $W^{*}(G, \sigma)$ are, respectively, the $C^{*}$ algebra and the von Neumann algebra generated by $\lambda_{\sigma}\left(\ell^{1}(G, \sigma)\right)$, or equivalently by $\lambda_{\sigma}(G)$.

If $\tau$ is similar with $\sigma$, then in all three cases, the operator algebras associated with $(G, \tau)$ and $(G, \sigma)$ are isomorphic.

Moreover, there is a natural one-to-one correspondence between the representations of $C^{*}(G, \sigma)$ and the $\sigma$-projective unitary representations of $G$. In particular, $\lambda_{\sigma}$ extends to a *-homomorphism of $C^{*}(G, \sigma)$ onto $C_{r}^{*}(G, \sigma)$. If $G$ is amenable, then $\lambda_{\sigma}$ is faithful. However, it is not known whether the converse holds unless $\sigma$ is trivial.

Following the work of Kleppner [13, an element $a$ in $G$ is called $\sigma$-regular if $\sigma(a, b)=\sigma(b, a)$ whenever $b$ commutes with $a$, or equivalently if

$$
U(a) U(b)=U(b) U(a)
$$

for all $b$ commuting with $a$ whenever $U$ is a $\sigma$-projective unitary representation of $G$. If $\sigma$ and $\tau$ are similar multipliers on $G$, it is easily seen that $a$ in $G$ is $\sigma$-regular if and only if it is $\tau$-regular. Furthermore, if $a$ is $\sigma$-regular, then $c a c^{-1}$ is $\sigma$-regular for all $c$ in $G$, and thus the notion of $\sigma$-regularity makes sense for conjugacy classes [13, Lemma 3]. The following theorem may now be deduced from [13, Lemma 4].

Theorem B. Let $\sigma$ be a multiplier on $G$. Then the following are equivalent:

(i) Every nontrivial $\sigma$-regular conjugacy class of $G$ is infinite.

(ii) $W^{*}(G, \sigma)$ is a factor.

Definition. We say that the pair $(G, \sigma)$ satisfies condition $K$ if (i) is satisfied.

If $G$ has the icc property, then $(G, \sigma)$ always satisfies condition K. If $G$ is abelian, or more generally, if all the conjugacy classes of $G$ are finite, then $(G, \sigma)$ satisfies condition K only if there are no nontrivial $\sigma$-regular elements in $G$.

Example 1.1. For $n \geq 2$, let $\mathbb{Z}_{n}$ denote the cyclic group of order $n$. Then $\mathcal{M}\left(\mathbb{Z}_{n} \times \mathbb{Z}_{n}\right) \cong \mathbb{Z}_{n}$ and its elements may be represented by multipliers $\sigma_{k}$ given by

$$
\sigma_{k}\left(\left(a_{1}, a_{2}\right),\left(b_{1}, b_{2}\right)\right)=e^{2 \pi i \frac{k}{n} a_{2} b_{1}}
$$

for $0 \leq k \leq n-1$. An element $a=\left(a_{1}, a_{2}\right)$ in $\mathbb{Z}_{n} \times \mathbb{Z}_{n}$ is $\sigma_{k}$-regular if and only if both $k a_{1}$ and $k a_{2}$ belong to $n \mathbb{Z}$. Therefore, $\left(\mathbb{Z}_{n} \times \mathbb{Z}_{n}, \sigma_{k}\right)$ satisfies condition $\mathrm{K}$ if and only if $k$ and $n$ are relatively prime, in which case we have

$$
C^{*}\left(\mathbb{Z}_{n} \times \mathbb{Z}_{n}, \sigma_{k}\right) \cong C_{r}^{*}\left(\mathbb{Z}_{n} \times \mathbb{Z}_{n}, \sigma_{k}\right)=W^{*}\left(\mathbb{Z}_{n} \times \mathbb{Z}_{n}, \sigma_{k}\right) \cong M_{n}(\mathbb{C}) .
$$

Example 1.2. It is well known that $\mathcal{M}\left(\mathbb{Z}^{n}\right) \cong \mathbb{T}^{\frac{1}{2} n(n-1)}$ and that the multipliers are, up to similarity, determined by

$$
\sigma_{\theta}(a, b)=e^{2 \pi i \sum_{1 \leq i<j \leq n} a_{i} t_{i j} b_{j}}
$$


for $\theta=\left(t_{12}, t_{13}, \ldots, t_{n-1, n}\right)$ in $[0,1)^{\frac{1}{2} n(n-1)}$. Note that the $C^{*}$-algebras associated with the pair $\left(\mathbb{Z}^{n}, \sigma_{\theta}\right), C^{*}\left(\mathbb{Z}^{n}, \sigma_{\theta}\right) \cong C_{r}^{*}\left(\mathbb{Z}^{n}, \sigma_{\theta}\right)$, are the noncommutative $n$-tori when $\theta$ is nonzero.

Furthermore, $\left(\mathbb{Z}^{n}, \sigma_{\theta}\right)$ satisfies condition $\mathrm{K}$ if there are no nontrivial $\sigma_{\theta^{-}}$ regular elements in $\mathbb{Z}^{n}$, that is, if there for all $a$ in $\mathbb{Z}^{n}$ exists $b$ in $\mathbb{Z}^{n}$ such that

$$
\sigma_{\theta}(a, b) \overline{\sigma_{\theta}(b, a)}=e^{2 \pi i \sum_{1 \leq i<j \leq n} t_{i j}\left(a_{i} b_{j}-b_{i} a_{j}\right)} \neq 1 .
$$

For $n=2$ and 3 we can give a good description of this property. Indeed, $\left(\mathbb{Z}^{2}, \sigma_{\theta}\right)$ satisfies condition $\mathrm{K}$ if and only if $\theta$ is irrational, and $\left(\mathbb{Z}^{3}, \sigma_{\theta}\right)$ satisfies condition $\mathrm{K}$ if and only if

$$
\operatorname{dim} \mathbb{Q}_{\theta}=3 \text { or } 4,
$$

where $\mathbb{Q}_{\theta}$ denotes the vector space over $\mathbb{Q}$ spanned by $\left\{1, t_{12}, t_{13}, t_{23}\right\}$.

For $n \geq 4$, the situation is more complicated. In particular, condition $\mathrm{K}$ on $\left(\mathbb{Z}^{n}, \sigma_{\theta}\right)$ does not only depend on the dimension of $\mathbb{Q}_{\theta}$. For example, if $t_{12}=t_{34}$ is some irrational number in $[0,1)$ and $t_{i j}=0$ elsewhere, then $\operatorname{dim} \mathbb{Q}_{\theta}=2$, and $\left(\mathbb{Z}^{4}, \sigma_{\theta}\right)$ satisfies condition $K$. On the other hand, if $t_{12}=t_{23}=t_{34}=1-t_{14}$ is some irrational number in $[0,1)$ and $t_{13}=t_{24}=0$, then $\operatorname{dim} \mathbb{Q}_{\theta}=2$, but it can be easily checked that $(1,1,1,1)$ in $\mathbb{Z}^{4}$ is $\sigma_{\theta}$-regular.

Example 1.3. For each natural number $n \geq 2$ let $G(n)$ be the group with presentation

$$
G(n)=\left\langle u_{i}, v_{j k}:\left[v_{j k}, v_{l m}\right]=\left[u_{i}, v_{j k}\right]=e,\left[u_{j}, u_{k}\right]=v_{j k}\right\rangle
$$

for $1 \leq i \leq n, 1 \leq j<k \leq n$ and $1 \leq l<m \leq n$. The group $G(n)$ is sometimes called the free nilpotent group of class 2 and rank $n$.

In a separate work, we will calculate the multipliers of $G(n)$ and show that

$$
\mathcal{M}(G(n)) \cong \mathbb{T}^{\frac{1}{3}(n-1) n(n+1)} .
$$

Note that $G(2)$ is isomorphic with the discrete Heisenberg group and this case is already investigated by Packer [20].

To describe our result in the case of $G(3)$, we first remark that $G(3)$ is isomorphic to the group with elements $a=\left(a_{1}, a_{2}, a_{3}, a_{4}, a_{5}, a_{6}\right)$, where all entries are integers, and with multiplication defined by

$$
a \cdot b=\left(a_{1}+b_{1}, a_{2}+b_{2}, a_{3}+b_{3}, a_{4}+b_{4}+a_{1} b_{2}, a_{5}+b_{5}+a_{1} b_{3}, a_{6}+b_{6}+a_{2} b_{3}\right) \text {. }
$$

For every $\mu$ in $\mathbb{T}^{8}$, the element $\left[\sigma_{\mu}\right]$ in $\mathcal{M}(G(3))$ may be represented by

$$
\begin{aligned}
\sigma_{\mu}(a, b)= & \mu_{13}^{b_{6} a_{1}-b_{3} a_{4}} \mu_{22}^{b_{5} a_{2}+b_{3}\left(a_{4}+a_{1} a_{2}\right)} \\
& \cdot \mu_{11}^{b_{4} a_{1}+\frac{1}{2} b_{2} a_{1}\left(a_{1}-1\right)} \mu_{21}^{a_{2}\left(b_{4}+a_{1} b_{2}\right)+\frac{1}{2} a_{1} b_{2}\left(b_{2}-1\right)} \\
& \cdot \mu_{12}^{b_{5} a_{1}+\frac{1}{2} b_{3} a_{1}\left(a_{1}-1\right)} \mu_{32}^{a_{3}\left(b_{5}+a_{1} b_{3}\right)+\frac{1}{2} a_{1} b_{3}\left(b_{3}-1\right)} \\
& \cdot \mu_{23}^{b_{6} a_{2}+\frac{1}{2} b_{3} a_{2}\left(a_{2}-1\right)} \mu_{33}^{a_{3}\left(b_{6}+a_{2} b_{3}\right)+\frac{1}{2} a_{2} b_{3}\left(b_{3}-1\right)}
\end{aligned}
$$

where $\mu_{i j} \in \mathbb{T}$.

The pair $\left(G(3), \sigma_{\mu}\right)$ satisfies condition $\mathrm{K}$ if and only if $G(3)$ has no nontrivial central $\sigma_{\mu}$-regular elements, that is, if for all $c=\left(0,0,0, c_{1}, c_{2}, c_{3}\right)$ in $Z(G(3))=$ $\mathbb{Z}^{3}$ there exists $a$ in $G(3)$ such that $\sigma_{\mu}(a, c) \overline{\sigma_{\mu}(c, a)} \neq 1$. 
Set $\mu_{31}=\mu_{13} \overline{\mu_{22}}$. One can then show that this holds if and only if for each nontrivial $c$ in $\mathbb{Z}^{3}$ there is some $i=1,2$ or 3 such that

$$
\prod_{1 \leq j \leq 3} \mu_{i j}^{c_{j}} \neq 1 .
$$

\section{Primeness and primitivity}

Henceforth, we fix a group $G$ and a multiplier $\sigma$ on $G$. Consider the right regular $\bar{\sigma}$-projective unitary representation $\rho_{\bar{\sigma}}$ of $G$ on $B\left(\ell^{2}(G)\right)$ defined by

$$
\left(\rho_{\bar{\sigma}}(a) \xi\right)(c)=\left(\xi *_{\bar{\sigma}} \delta_{a}^{*}\right)(c)=\overline{\sigma(c, a)} \xi(c a)
$$

To simplify notation in what follows, we write just $\bar{\rho}$ and $\lambda$ for $\rho_{\bar{\sigma}}$ and $\lambda_{\sigma}$. It is straightforward to see that $\lambda(a)$ commutes with $\bar{\rho}(b)$ for all $a, b$ in $G$, that is, $W^{*}(G, \sigma)$ is contained in $\bar{\rho}(G)^{\prime}$. In fact, it is well known that $W^{*}(G, \sigma)=\bar{\rho}(G)^{\prime}$. Moreover,

$$
(\lambda(a) \bar{\rho}(a) \xi)(c)=\overline{\sigma\left(a^{-1}, c\right)} \sigma\left(a^{-1} c a, a^{-1}\right) \xi\left(a^{-1} c a\right)
$$

for all $a, c \in G$ and all $\xi \in \ell^{2}(G)$. In particular,

$$
\lambda(a) \bar{\rho}(a) \delta_{e}=\bar{\rho}(a) \lambda(a) \delta_{e}=\delta_{e}
$$

for all $a \in G$.

Remark 2.1. The vector $\delta_{e}$ is clearly cyclic for $W^{*}(G, \sigma)$. It is also separating. Indeed, if $x \delta_{e}=0$, then

$$
x \delta_{a}=x \lambda(a) \delta_{e}=x \bar{\rho}(a)^{*} \delta_{e}=\bar{\rho}(a)^{*} x \delta_{e}=0
$$

for all $a \in G$. Moreover, the state $\varphi$ given by $\varphi(x)=\left\langle x \delta_{e}, \delta_{e}\right\rangle$ is a faithful trace on $W^{*}(G, \sigma)$. Thus, $W^{*}(G, \sigma)$ is finite and is therefore a $\mathrm{II}_{1}$ factor whenever $G$ is infinite and $(G, \sigma)$ satisfies condition $\mathrm{K}$, according to Theorem B

Lemma 2.2. Let $T$ be an operator in $W^{*}(G, \sigma)$ and set $f_{T}=T \delta_{e}$. Then the following are equivalent:

(i) $T$ belongs to the center of $W^{*}(G, \sigma)$.

(ii) $f_{T}\left(a c a^{-1}\right)=\sigma(a, c) \overline{\sigma\left(a c a^{-1}, a\right)} f_{T}(c)$ for all $a, c \in G$.

Moreover, $f_{T}$ can be nonzero only on the finite conjugacy classes.

Proof. The operator $T$ belongs to the center of $W^{*}(G, \sigma)$ if and only if $T=$ $\lambda(a) T \lambda(a)^{*}$ for all $a \in G$. Since, by Remark 2.1, $\delta_{e}$ is separating for $W^{*}(G, \sigma)$, this is equivalent to $f_{T}=\lambda(a) T \lambda(a)^{*} \delta_{e}$ for all $a \in G$. By (4) we have

$$
\lambda(a) T \lambda(a)^{*} \delta_{e}=\lambda(a) T \bar{\rho}(a) \delta_{e}=\lambda(a) \bar{\rho}(a) T \delta_{e}=\lambda(a) \bar{\rho}(a) f_{T}
$$

for all $a \in G$. Thus $T$ belongs to the center if and only if $f_{T}=\lambda(a) \bar{\rho}(a) f_{T}$ for all $a \in G$ and the desired equivalence now follows from (3). If a function $f$ satisfies (ii), then $|f|$ is constant on conjugacy classes. Since $f_{T}$ belongs to $\ell^{2}(G)$, it can be nonzero only on the finite conjugacy classes. 
Remark 2.3. Lemma 2.2 is proved in [13, Theorem 1]. However, the proof provided above is shorter. Lemma 2.4 below is proved in [13, Lemma 2] in the case where $C$ is a single point. Also, note that we do not restrict to normalized multipliers as in [13].

Lemma 2.4. Let $C$ be a conjugacy class of $G$. Then following are equivalent:

(i) $C$ is $\sigma$-regular.

(ii) There is a function $f: G \rightarrow \mathbb{C}$ satisfying:

1. $f(c) \neq 0$ for all $c \in C$.

2. $f\left(a c a^{-1}\right)=\sigma(a, c) \overline{\sigma\left(a c a^{-1}, a\right)} f(c)$ for all $c \in C$ and all $a \in G$.

Moreover, $f$ can be chosen in $\ell^{2}(G)$ if and only if $C$ is finite.

Proof. (ii) $\Rightarrow($ i): Suppose $c$ belongs to $C$ and that $a$ commutes with $c$. Then there is a function $f: G \rightarrow \mathbb{C}$ satisfying $0 \neq f(c)=\sigma(a, c) \overline{\sigma(c, a)} f(c)$. Hence $\sigma(a, c)=\sigma(c, a)$, so $c$ is $\sigma$-regular.

(i) $\Rightarrow$ (ii): This clearly holds if $C$ is trivial, so suppose $C$ is nontrivial and $\sigma$-regular and fix an element $c$ in $C$. Define a function $f: G \rightarrow \mathbb{C}$ by

$$
f(x)= \begin{cases}\sigma(a, c) \overline{\sigma\left(a c a^{-1}, a\right)} & \text { if } x \in C, \quad x=a c a^{-1} \text { for some } a \in G \\ 0 & \text { if } x \notin C\end{cases}
$$

First we show that $f$ is well-defined, so assume $a c a^{-1}=b c b^{-1}$, and note that

$$
\begin{aligned}
\sigma\left(a^{-1}, a c a^{-1}\right) \sigma\left(c a^{-1}, b\right) & =\sigma\left(a^{-1}, a c a^{-1} b\right) \sigma\left(a c a^{-1}, b\right) \\
& =\sigma\left(a^{-1}, b c\right) \sigma\left(b c b^{-1}, b\right)
\end{aligned}
$$

As $c$ is $\sigma$-regular and commutes with $a^{-1} b, \sigma\left(a^{-1} b, c\right)=\sigma\left(c, a^{-1} b\right)$. Thus

$$
\begin{aligned}
\sigma\left(c, a^{-1}\right) \sigma\left(c a^{-1}, b\right) & =\sigma\left(c, a^{-1} b\right) \sigma\left(a^{-1}, b\right) \\
& =\sigma\left(a^{-1}, b\right) \sigma\left(a^{-1} b, c\right)=\sigma\left(a^{-1}, b c\right) \sigma(b, c) .
\end{aligned}
$$

Together, we get from these equations that

$$
\sigma\left(a^{-1}, a c a^{-1}\right) \sigma(b, c)=\sigma\left(c, a^{-1}\right) \sigma\left(b c b^{-1}, b\right) .
$$

Finally, the two identities

$$
\begin{gathered}
\sigma\left(a^{-1}, a c a^{-1}\right) \sigma\left(c a^{-1}, a\right)=\sigma\left(a^{-1}, a c\right) \sigma\left(a c a^{-1}, a\right) \\
\sigma\left(c, a^{-1}\right) \sigma\left(c a^{-1}, a\right)=\sigma\left(a^{-1}, a\right)=\sigma\left(a^{-1}, a c\right) \sigma(a, c)
\end{gathered}
$$

give that

$$
\sigma\left(a^{-1}, a c a^{-1}\right) \sigma(a, c)=\sigma\left(c, a^{-1}\right) \sigma\left(a c a^{-1}, a\right) .
$$

Combining (5) and (6) we get that

$$
\sigma(a, c) \overline{\sigma\left(a c a^{-1}, a\right)}=\sigma(b, c) \overline{\sigma\left(b c b^{-1}, b\right)} .
$$

Hence $f$ is well-defined, so $f\left(a c a^{-1}\right)=f\left(b c b^{-1}\right)$. 
It is easily seen that $|f(x)|=1$ for all $x$ in $C$. In fact, if $f$ is any function satisfying (ii), then $|f|$ must be constant and nonzero on $C$, hence $f$ belongs to $\ell^{2}(G)$ if and only if $C$ is finite.

In particular, $f(c)=1$ in our case, so $f$ satisfies part 2 of (ii) for the chosen $c$ in $C$. It remains to show that $f$ satisfies part 2 of (ii) for all other $x$ in $C$. Suppose $x$ is an element of $C$, that is, there exists $b$ in $G$ such that $x=b c b^{-1}$. Note first that

$$
f(x)=f\left(b c b^{-1}\right)=\sigma(b, c) \overline{\sigma\left(b c b^{-1}, b\right)}=\sigma(b, c) \overline{\sigma(x, b)} .
$$

Next,

$$
\begin{aligned}
\sigma\left(a x a^{-1}, a\right) \sigma(a x, b) \sigma(a b, c) & =\sigma\left(a x a^{-1}, a b\right) \sigma(a, b) \sigma(a b, c) \\
& =\sigma\left(a x a^{-1}, a b\right) \sigma(a, b c) \sigma(b, c),
\end{aligned}
$$

which, since $x b=b c$, gives that

$$
\begin{aligned}
\sigma(a, x) \overline{\sigma(x, b)} & =\sigma(a, x b) \overline{\sigma(a x, b)}=\sigma(a, b c) \overline{\sigma(a x, b)} \\
& =\sigma\left(a x a^{-1}, a\right) \sigma(a b, c) \overline{\sigma\left(a x a^{-1}, a b\right) \sigma(b, c) .}
\end{aligned}
$$

Hence

$$
\begin{aligned}
f\left(a x a^{-1}\right) & =f\left(a b c b^{-1} a^{-1}\right)=\sigma(a b, c) \overline{\sigma\left(a b c b^{-1} a^{-1}, a b\right)} \\
& =\sigma(a b, c) \overline{\sigma\left(a x a^{-1}, a b\right)}=\sigma(a, x) \overline{\sigma\left(a x a^{-1}, a\right)} \sigma(b, c) \overline{\sigma(x, b)} \\
& =\sigma(a, x) \overline{\sigma\left(a x a^{-1}, a\right)} f(x) .
\end{aligned}
$$

Before stating the main theorem, we recall two results which are part of the folklore of operator algebras. The first can be shown as sketched in the proof of [18, Proposition 2.3], while the second is a rather easy consequence of Urysohn's Lemma. Remark that together these two results imply that if $A$ is von Neumann algebra, then $A$ is prime if and only if it is a factor.

Proposition 2.5. If $A$ is a concrete unital $C^{*}$-algebra and its bicommutant $A^{\prime \prime}$ is a factor, then $A$ is prime.

Proposition 2.6. Every prime $C^{*}$-algebra has trivial center.

Theorem 2.7. The following conditions are equivalent:

(i) $(G, \sigma)$ satisfies condition $K$.

(ii) $W^{*}(G, \sigma)$ is a factor.

(iii) $C_{r}^{*}(G, \sigma)$ is prime.

(iv) $C_{r}^{*}(G, \sigma)$ has trivial center. 
Proof. For completeness, we include the few lines required to prove (i) $\Rightarrow$ (ii): Suppose $(G, \sigma)$ satisfies condition $\mathrm{K}$ and let $T$ belong to the center of $W^{*}(G, \sigma)$. By Lemma 2.2 and Lemma 2.4, $f_{T}$ can be nonzero only on the finite $\sigma$-regular conjugacy classes, hence on $\{e\}$. So $T \delta_{e}=f_{T}(e) \delta_{e}$, thus $T=f_{T}(e) I$ as $\delta_{e}$ is separating for $W^{*}(G, \sigma)$ by Remark 2.1.

The implications (ii) $\Rightarrow$ (iii) $\Rightarrow$ (iv) follow from Proposition 2.5] and 2.6.

(iv) $\Rightarrow$ (i): Suppose $C$ is a finite nontrivial $\sigma$-regular conjugacy class of $G$. Let $f$ be a function satisfying part (ii) of Lemma 2.4 and define the operator $T=\sum_{c \in C} f(c) \lambda(c)$. Then $T$ belongs to the center of $C_{r}^{*}(G, \sigma)$. Indeed,

$$
\begin{aligned}
\lambda(a) T \lambda(a)^{*} & =\sum_{c \in C} f(c) \lambda(a) \lambda(c) \lambda(a)^{*} \\
& =\sum_{c \in C} f(c) \sigma(a, c) \overline{\sigma\left(a c a^{-1}, a\right)} \lambda\left(a c a^{-1}\right) \\
& =\sum_{b \in a C a^{-1}} f\left(a^{-1} b a\right) \sigma\left(a, a^{-1} b a\right) \overline{\sigma(b, a)} \lambda(b) \\
& =\sum_{b \in C} f\left(a^{-1} b a\right) \overline{\sigma\left(a^{-1}, b\right)} \sigma\left(a^{-1} b a, a^{-1}\right) \lambda(b) \\
& =\sum_{b \in C} f(b) \lambda(b)=T
\end{aligned}
$$

for all $a \in G$, where the identity (6) is used to get the fourth equality.

The proof of the following corollary goes along the same lines as the one given in [18, Proposition 2.1] in the untwisted case.

Corollary 2.8. If $C^{*}(G, \sigma)$ is prime, then $(G, \sigma)$ satisfies condition $K$.

Proof. Observe that the set $\{\lambda(a)\}_{a \in G}$ is linear independent in $C_{r}^{*}(G, \sigma)$, and the universal property of $C^{*}(G, \sigma)$ ensures that there is a surjective *-homomorphism $C^{*}(G, \sigma) \rightarrow C_{r}^{*}(G, \sigma)$ mapping $i_{G}(a)$ to $\lambda(a)$. Hence, $\left\{i_{G}(a)\right\}_{a \in G}$ is also linear independent and has dense span in $C^{*}(G, \sigma)$.

Therefore, the result follows by replacing $i_{G}$ with $\lambda$ in the proof of Theorem [2.7, and repeating the argument for (iii) $\Rightarrow$ (iv) $\Rightarrow$ (i) word by word.

Remark 2.9. In general, the center of $C^{*}(G, \sigma)$ is not easily determined.

However, a slightly stronger version of Corollary 2.8 is known in the untwisted case. If $C^{*}(G)$ has trivial center, then $G / H$ is icc whenever $H$ is a normal subgroup of $G$ satisfying Kazhdan's property T (see e.g. 14]).

Corollary 2.10 ([21, Proposition 1.4]). Assume $G$ is countable and amenable. Then the following conditions are equivalent:

(i) $(G, \sigma)$ satisfies condition $K$.

(ii) $C^{*}(G, \sigma)$ is primitive.

Proof. If $(G, \sigma)$ satisfies condition $\mathrm{K}$, then $C_{r}^{*}(G, \sigma)$ is prime by Theorem 2.7. As $G$ is countable, $C_{r}^{*}(G, \sigma)$ is separable and hence primitive by Dixmier's result. Now, the amenability of $G$ implies that $C^{*}(G, \sigma) \cong C_{r}^{*}(G, \sigma)$, so $C^{*}(G, \sigma)$ is also primitive. Finally, (ii) always implies (i) by Corollary 2.8 . 
Remark 2.11. Condition $\mathrm{K}$ on $(G, \sigma)$ does not imply primeness or primitivity of $C^{*}(G, \sigma)$ in general. To see this, let $G=\operatorname{SL}(3, \mathbb{Z})$ and $\sigma=1$. Then, $G$ is countable, icc and satisfies Kazhdan's property T. In particular, $G$ is nonamenable. As explained in [4, Proposition 2.5], $C^{*}(G)$ is not primitive.

On the other hand, I don't know any example of an uncountable and amenable group such that (i) holds, but not (ii).

Remark 2.12. If $G$ is countable and nilpotent, then condition $\mathrm{K}$ on $(G, \sigma)$ is actually equivalent to simplicity of $C^{*}(G, \sigma)$ [21, Proposition 1.7]. The same is also true if $G$ is finite.

However, this does not hold for all countable, amenable groups. For example, if $G$ is the group of all finite permutations on a countably infinite set, then $G$ is countable, amenable and icc, so $C^{*}(G)$ is primitive and nonsimple.

Remark 2.13. Note that $C_{r}^{*}(\mathrm{SL}(3, \mathbb{Z}))$ is known to be simple [5], so Remark 2.11] and 2.12 show that primitivity of a full twisted group $C^{*}$-algebra is in general unrelated to simplicity of the corresponding reduced twisted group $C^{*}$-algebra.

Proposition 2.14. The following conditions are equivalent:

(i) $G$ is amenable.

(ii) $C^{*}(G, \sigma)$ is nuclear.

(iii) $C_{r}^{*}(G, \sigma)$ is nuclear.

(iv) $W^{*}(G, \sigma)$ is injective.

Proof. This is well known in the untwisted case. The result in the twisted case appeared in a preprint by Bédos and Conti [2], but was left out in the final version. For the convenience of the reader we repeat the argument. First, (i) $\Rightarrow$ (ii) follows from [22, Corollary 3.9]. The implication (ii) $\Rightarrow$ (iii) holds since every quotient of a nuclear $C^{*}$-algebra is nuclear. Moreover, the von Neumann algebra generated by a nuclear $C^{*}$-algebra is injective, hence (iii) $\Rightarrow$ (iv). Finally, if $W^{*}(G, \sigma)$ is injective, it has a hypertrace and thus $G$ is amenable by [1, Corollary 1.7], so (iv) $\Rightarrow$ (i).

According to 8, all injective $\mathrm{II}_{1}$ factors acting on a separable Hilbert space are isomorphic to the hyperfinite $\mathrm{II}_{1}$ factor. Hence, we get the following corollary.

Corollary 2.15. Assume $G$ is countably infinite. Then the following conditions are equivalent:

(i) $G$ is amenable and $(G, \sigma)$ satisfies condition $K$.

(ii) $C^{*}(G, \sigma)$ is nuclear and primitive.

(iii) $C_{r}^{*}(G, \sigma)$ is nuclear and primitive.

(iv) $W^{*}(G, \sigma)$ is the hyperfinite $I I_{1}$ factor. 


\section{Direct products}

Let $G_{1}$ and $G_{2}$ be two groups. A function $f: G_{1} \times G_{2} \rightarrow \mathbb{T}$ is called a bihomomorphism if

$$
f\left(a_{1} b_{1}, a_{2}\right)=f\left(a_{1}, a_{2}\right) f\left(b_{1}, a_{2}\right) \quad \text { and } \quad f\left(a_{1}, a_{2} b_{2}\right)=f\left(a_{1}, a_{2}\right) f\left(a_{1}, b_{2}\right)
$$

for all $a_{1}, b_{1} \in G_{1}$ and $a_{2}, b_{2} \in G_{2}$. Let $B\left(G_{1}, G_{2}\right)$ denote the set of bihomomorphisms $G_{1} \times G_{2} \rightarrow \mathbb{T}$. This is a group under pointwise multiplication and is isomorphic with $\operatorname{Hom}\left(G_{1}, \operatorname{Hom}\left(G_{2}, \mathbb{T}\right)\right)$.

It is well known (see e.g. [15) that the Schur multiplier of $G_{1} \times G_{2}$ decomposes as

$$
\mathcal{M}\left(G_{1} \times G_{2}\right) \cong \mathcal{M}\left(G_{1}\right) \oplus \mathcal{M}\left(G_{2}\right) \oplus B\left(G_{1}, G_{2}\right) .
$$

We will only need to know the following. Let $\left(\sigma_{1}, \sigma_{2}, f\right)$ be a triple where $\sigma_{1}$ and $\sigma_{2}$ are multipliers on $G_{1}$ and $G_{2}$, respectively, and $f$ belongs to $B\left(G_{1}, G_{2}\right)$. Then we can define a multiplier $\sigma$ on $G_{1} \times G_{2}$ by

$$
\sigma\left(\left(a_{1}, a_{2}\right),\left(b_{1}, b_{2}\right)\right)=\sigma_{1}\left(a_{1}, b_{1}\right) \sigma_{2}\left(a_{2}, b_{2}\right) f\left(b_{1}, a_{2}\right)
$$

for $a_{1}, b_{1} \in G_{1}$ and $a_{2}, b_{2} \in G_{2}$, and it can be shown that every multiplier on $G_{1} \times G_{2}$ is similar to such a $\sigma$. When $\sigma$ is a multiplier on $G_{1} \times G_{2}$, we let $\sigma_{1}$ be the multiplier on $G_{1}$ defined by

$$
\sigma_{1}\left(a_{1}, b_{1}\right)=\sigma\left(\left(a_{1}, e\right),\left(b_{1}, e\right)\right)
$$

for $a_{1}, b_{1} \in G_{1}$ and call it the restriction of $\sigma$ to $G_{1}$. Similarly we can define the restriction $\sigma_{2}$ of $\sigma$ to $G_{2}$.

Henceforth, we fix two groups $G_{1}$ and $G_{2}$, multipliers $\sigma_{1}$ on $G_{1}$ and $\sigma_{2}$ on $G_{2}$, and a bihomomorphism $f$ in $B\left(G_{1}, G_{2}\right)$. We set $G=G_{1} \times G_{2}$ and let $\sigma$ be the multiplier on $G$ defined by (77). Moreover, we write $\sigma=\sigma_{1} \times \sigma_{2}$ if $f=1$.

It is convenient to record the identity

$$
\sigma(a, b) \overline{\sigma(b, a)} \cdot f\left(a_{1}, b_{2}\right) \overline{f\left(b_{1}, a_{2}\right)}=\sigma_{1}\left(a_{1}, b_{1}\right) \overline{\sigma_{1}\left(b_{1}, a_{1}\right)} \cdot \sigma_{2}\left(a_{2}, b_{2}\right) \overline{\sigma_{2}\left(b_{2}, a_{2}\right)}
$$

which follows directly from (7). Note also that $C$ is a conjugacy class of $G$ if and only if $C=C_{1} \times C_{2}$ where $C_{1}$ and $C_{2}$ are conjugacy classes of $G_{1}$ and $G_{2}$, respectively.

Proposition 3.1. The following are equivalent:

(i) $C_{r}^{*}(G, \sigma)$ is prime.

(ii) For every finite nontrivial conjugacy class $C$ of $G$, there exist a $=\left(a_{1}, a_{2}\right)$ in $C$ and $b=\left(b_{1}, b_{2}\right)$ in $G$ such that at least one of these conditions hold:

1. $a_{1} b_{1}=b_{1} a_{1}$ and $f\left(b_{1}, a_{2}\right) \neq \overline{\sigma_{1}\left(a_{1}, b_{1}\right)} \sigma_{1}\left(b_{1}, a_{1}\right)$.

2. $a_{2} b_{2}=b_{2} a_{2}$ and $f\left(a_{1}, b_{2}\right) \neq \sigma_{2}\left(a_{2}, b_{2}\right) \overline{\sigma_{2}\left(b_{2}, a_{2}\right)}$.

Proof. Suppose that condition (ii) does not hold. Then there is a finite nontrivial conjugacy class $C$ such that both 1 . and 2 . fail for all $a$ in $C$ and $b$ in $G$. Hence, $f\left(b_{1}, a_{2}\right)=\overline{\sigma_{1}\left(a_{1}, b_{1}\right)} \sigma_{1}\left(b_{1}, a_{1}\right)$ and $f\left(a_{1}, b_{2}\right)=\sigma_{2}\left(a_{2}, b_{2}\right) \overline{\sigma_{2}\left(b_{2}, a_{2}\right)}$ whenever $a=\left(a_{1}, a_{2}\right)$ is in $C, b=\left(b_{1}, b_{2}\right)$ in $G$ and $b$ commutes with $a$. Then $C$ is 
$\sigma$-regular by (8), and therefore $(G, \sigma)$ does not satisfy condition $\mathrm{K}$, that is, $C_{r}^{*}(G, \sigma)$ is not prime by Theorem 2.7. Thus, (i) $\Rightarrow$ (ii).

Conversely, assume that $(G, \sigma)$ does not satisfy condition $\mathrm{K}$ and let $C=C_{1} \times$ $C_{2}$ be a finite nontrivial $\sigma$-regular conjugacy class of $G$. If $b_{1}$ in $G_{1}$ commutes with $a_{1}$ in $C_{1}$, then $\left(b_{1}, e\right)$ commutes with $\left(a_{1}, a_{2}\right)$ for every $a_{2}$ in $C_{2}$. Hence, the $\sigma$-regularity of $C$ and identity (8) give that

$$
f\left(b_{1}, a_{2}\right)=\overline{\sigma_{1}\left(a_{1}, b_{1}\right)} \sigma_{1}\left(b_{1}, a_{1}\right)
$$

whenever $a$ belongs to $C$ and $b_{1}$ in $G_{1}$ commutes with $a_{1}$. Similarly,

$$
f\left(a_{1}, b_{2}\right)=\sigma_{2}\left(a_{2}, b_{2}\right) \overline{\sigma_{2}\left(b_{2}, a_{2}\right)}
$$

whenever $b_{2}$ in $G_{2}$ commutes with $a_{2}$. It follows that for all $a$ in $C$ and $b$ in $G$, both 1. and 2. fail to hold, hence condition (ii) is not satisfied.

Remark 3.2. Let $G_{1}$ and $G_{2}$ be abelian and assume that $\sigma_{1}$ and $\sigma_{2}$ are trivial. Condition (ii) of Proposition 3.1 then says that for all nontrivial $\left(a_{1}, a_{2}\right)$ in $G$ there exists $\left(b_{1}, b_{2}\right)$ in $G$ such that $f\left(a_{1}, b_{2}\right) \neq 1$ or $f\left(b_{1}, a_{2}\right) \neq 1$. If this holds, $\sigma$ is called nondegenerate and it was first shown by Slawny [23, Theorem 3.7] that $C^{*}(G, \sigma) \cong C_{r}^{*}(G, \sigma)$ is simple in this case.

Lemma 3.3. Let $a=\left(a_{1}, a_{2}\right)$ be an element in $G$. If two of the following conditions hold, then all three hold:

(i) a is $\sigma$-regular.

(ii) $a_{i}$ is $\sigma_{i}$-regular for both $i=1$ and 2 .

(iii) $f\left(a_{1}, b_{2}\right)=f\left(b_{1}, a_{2}\right)$ whenever $b=\left(b_{1}, b_{2}\right)$ commutes with $a$.

Moreover, (iii) is equivalent to:

(iv) $f\left(a_{1}, b_{2}\right)=f\left(b_{1}, a_{2}\right)=1$ whenever $b=\left(b_{1}, b_{2}\right)$ commutes with $a$.

Proof. Suppose that (ii) holds and pick any $b=\left(b_{1}, b_{2}\right)$ in $G$. Then it follows readily from (8) that (i) holds if and only if (iii) holds.

Next, assume that (iii) holds and let $b=\left(b_{1}, b_{2}\right)$ commute with $a$. Then $b^{\prime}=\left(b_{1}, e\right)$ also commutes with $a$, so $1=f\left(a_{1}, e\right)=f\left(b_{1}, a_{2}\right)$. Similarly, we get $f\left(a_{1}, b_{2}\right)=1$ and thus (iv) holds.

Suppose finally that (i) and (iii) hold and pick an element $b=\left(b_{1}, b_{2}\right)$ in $G$ that commutes with $a$. As (iv) also holds, we have that $f\left(b_{1}, a_{2}\right)=1$. By applying (8) with $b^{\prime}=\left(b_{1}, e\right)$, we see that $a_{1}$ is $\sigma_{1}$-regular. Similarly, $f\left(a_{1}, b_{2}\right)=$ 1 and $a_{2}$ is $\sigma_{2}$-regular.

Corollary 3.4. Let $C=C_{1} \times C_{2}$ be a conjugacy class of $G$. Suppose there is some $a=\left(a_{1}, a_{2}\right)$ in $C$ such that $f\left(a_{1}, b_{2}\right)=f\left(b_{1}, a_{2}\right)$ whenever $b=\left(b_{1}, b_{2}\right)$ commutes with $a$. Then the following are equivalent:

(i) $C$ is a finite nontrivial $\sigma$-regular conjugacy class of $G$.

(ii) $C_{i}$ is a finite $\sigma_{i}$-regular conjugacy class of $G_{i}$ for both $i=1$ and 2 and at least one of $C_{1}$ and $C_{2}$ is nontrivial. 
Corollary 3.5. Suppose both $C_{r}^{*}\left(G_{1}, \sigma_{1}\right)$ and $C_{r}^{*}\left(G_{2}, \sigma_{2}\right)$ are prime. Let $a=$ $\left(a_{1}, a_{2}\right)$ be such that $f\left(a_{1}, b_{2}\right)=f\left(b_{1}, a_{2}\right)$ whenever $b=\left(b_{1}, b_{2}\right)$ commutes with a. Then at most one of the following two conditions hold:

(i) a is $\sigma$-regular.

(ii) a belongs to a finite nontrivial conjugacy class of $G$.

Corollary 3.6. Suppose $f\left(a_{1}, b_{2}\right)=f\left(b_{1}, a_{2}\right)$ whenever $a=\left(a_{1}, a_{2}\right)$ is $\sigma$-regular and $b=\left(b_{1}, b_{2}\right)$ commutes with $a$. Then $C_{r}^{*}(G, \sigma)$ is prime if both $C_{r}^{*}\left(G_{1}, \sigma_{1}\right)$ and $C_{r}^{*}\left(G_{2}, \sigma_{2}\right)$ are prime.

Remark 3.7. In general, primeness of $C_{r}^{*}(G, \sigma)$ does not imply primeness of either $C_{r}^{*}\left(G_{1}, \sigma_{1}\right)$ or $C_{r}^{*}\left(G_{2}, \sigma_{2}\right)$. For example, if $G_{1}=G_{2}=\mathbb{Z}$, then $C^{*}(G, \sigma)$ can be simple even if both $\sigma_{1}$ and $\sigma_{2}$ are trivial.

Also, $C_{r}^{*}(G, \sigma)$ can be nonprime even if both $C^{*}\left(G_{1}, \sigma_{1}\right)$ and $C^{*}\left(G_{2}, \sigma_{2}\right)$ are simple. To see this, let $G_{1}=G_{2}=\mathbb{Z}^{2}$ and consider the case described in the last part of Example 1.2.

Proposition 3.8. Suppose $f\left(c_{1}, c_{2}\right)=1$ whenever $c_{i}$ belongs to a finite conjugacy class of $G_{i}$ for either $i=1$ or 2 . Then $C_{r}^{*}(G, \sigma)$ is prime if and only if both $C_{r}^{*}\left(G_{1}, \sigma_{1}\right)$ and $C_{r}^{*}\left(G_{2}, \sigma_{2}\right)$ are prime.

In particular, this holds when $\sigma=\sigma_{1} \times \sigma_{2}$.

Proof. Suppose $C_{r}^{*}(G, \sigma)$ is prime and $C_{1}$ is a finite $\sigma_{1}$-regular conjugacy class of $G_{1}$. Then $C_{1} \times\{e\}$ is $\sigma$-regular by Corollary 3.4 so $C_{1}=\{e\}$ and hence $C_{r}^{*}\left(G_{1}, \sigma_{1}\right)$ is prime. Similarly we get that $C_{r}^{*}\left(G_{2}, \sigma_{2}\right)$ is prime.

The converse follows directly from Corollary 3.5 .

Remark 3.9. Assume that $\sigma=\sigma_{1} \times \sigma_{2}$. Then $C_{r}^{*}(G, \sigma)$ is simple if and only both $C_{r}^{*}\left(G_{1}, \sigma_{1}\right)$ and $C_{r}^{*}\left(G_{2}, \sigma_{2}\right)$ are simple. Indeed, note that the map $\lambda_{\sigma}\left(a_{1}, a_{2}\right) \mapsto \lambda_{\sigma_{1}}\left(a_{1}\right) \otimes \lambda_{\sigma_{2}}\left(a_{2}\right)$ induces an isomorphism

$$
C_{r}^{*}(G, \sigma) \cong C_{r}^{*}\left(G_{1}, \sigma_{1}\right) \otimes_{\min } C_{r}^{*}\left(G_{2}, \sigma_{2}\right) .
$$

The result now follows from the fact that a spatial tensor product of two $C^{*}$ algebras is simple if and only if both involved $C^{*}$-algebras are simple (see [12, 11.5.5-6]).

The only positive result on primitivity so far in this paper concerns countable, amenable groups. However, Corollary 2.10 relies on Dixmier's result that is not constructive in the sense that it does not give a procedure to construct an explicit faithful irreducible representation.

In some cases, one may construct faithful irreducible representations of $C^{*}(G, \sigma)$ through an inducing process on representations of $C^{*}\left(G_{1}, \sigma_{1}\right)$.

Theorem 3.10. Assume that $G_{2}$ is amenable. Suppose there exists a faithful irreducible representation $\pi$ of $C^{*}\left(G_{1}, \sigma_{1}\right)$ such that for any given nontrivial $a_{2}$ in $G_{2}$, there exists $a_{1}$ in $G_{1}$ such that

$$
f\left(a_{1}, a_{2}\right) \pi\left(i_{G_{1}}\left(a_{1}\right)\right) \not \pi \pi\left(i_{G_{1}}\left(a_{1}\right)\right) .
$$

Then $C^{*}(G, \sigma)$ is primitive. 
Proof. Recall that there is a twisted action $(\alpha, \omega)$ of $G_{2}$ on $A=C^{*}\left(G_{1}, \sigma_{1}\right)$ satisfying (see e.g. 24])

$$
\begin{aligned}
\alpha_{a_{2}}\left(i_{G_{1}}\left(a_{1}\right)\right) & =\overline{f\left(a_{1}, a_{2}\right)} i_{G_{1}}\left(a_{1}\right), \\
\omega\left(a_{2}, b_{2}\right) & =\sigma_{2}\left(a_{2}, b_{2}\right) .
\end{aligned}
$$

Hence, there is also a natural action of $G_{2}$ on the set $\widehat{A}^{0}$ of equivalence classes of faithful irreducible representations of $A$ given by

$$
a_{2} \cdot[\psi]=\left[\psi \circ \alpha_{a_{2}^{-1}}\right] .
$$

For any given nontrivial $a_{2}$ in $G_{2}$, the assumptions on $\pi$ gives that

$$
\pi \circ \alpha_{a_{2}^{-1}}\left(i_{G_{1}}\left(a_{1}\right)\right)=f\left(a_{1}, a_{2}\right) \pi\left(i_{G_{1}}\left(a_{1}\right)\right) \nsucc \pi\left(i_{G_{1}}\left(a_{1}\right)\right)
$$

for some $a_{1}$ in $G_{1}$. Hence

$$
a_{2} \cdot[\pi] \neq[\pi]
$$

for all nontrivial $a_{2}$ in $G_{2}$. In other words, $[\pi]$ is a free point for this action. The conclusion follows from [4, Theorem 2.1].

Example 3.11. Let $G=\mathbb{F}_{2} \times \mathbb{Z}$ and let $u, v$ be the generators of $\mathbb{F}_{2}$. Since $\mathcal{M}\left(\mathbb{F}_{2}\right)=\mathcal{M}(\mathbb{Z})=\{1\}$, every multiplier on $G$ is, up to similarity, determined by a bihomomorphism $f: \mathbb{F}_{2} \times \mathbb{Z} \rightarrow \mathbb{T}$. Moreover, $f$ is determined by its values on the generators, that is, by $f(u, 1)$ and $f(v, 1)$. Let $\sigma$ be the multiplier on $G$ defined by these two numbers, say $\mu$ and $\nu$. We remark that

$$
C^{*}(G, \sigma) \cong C^{*}\left(\mathbb{F}_{2}\right) \rtimes_{\alpha} \mathbb{Z}
$$

where $\alpha$ is determined by $\alpha_{k}\left(i_{\mathbb{F}_{2}}(x)\right)=\overline{f(x, k)} i_{\mathbb{F}_{2}}(x)$ for $x \in \mathbb{F}_{2}$ and $k \in \mathbb{Z}$.

Assume $\mu$ is nontorsion and let $A=C^{*}\left(\mathbb{F}_{2}\right)$ sit inside $B(\mathcal{H})$ for some separable Hilbert space $\mathcal{H}$. Let $U=i_{\mathbb{F}_{2}}(u)$ and $V=i_{\mathbb{F}_{2}}(v)$ be the two unitaries in $B(\mathcal{H})$ generating $A$. Now, proceeding as Choi in [7, Lemma 4], there is an operator $D$ for which $U-D$ is compact and such that the following hold; with respect to a suitable basis on $\mathcal{H}, D$ is diagonal with diagonal entries $\left\{z_{i}\right\}_{i=1}^{\infty}$ satisfying $\left|z_{i}\right|=1$ for all $i, z_{1}=1, z_{i} \neq z_{j}$ if $i \neq j$ and $z_{i} \notin\left\{\mu^{k}: k \in \mathbb{Z}\right\}$ when $i \geq 2$.

Using [7, Lemma 5], we can find a compact perturbation $E$ of $V$ which is a unitary operator having no common nontrivial invariant subspace with $D$. Then, as explained in [7, Theorem 6], the map $U \mapsto D, V \mapsto E$ defines a faithful and irreducible representation $\pi$ of $A$ on $\mathcal{H}$.

Now we have

$$
\pi \circ \alpha_{k^{-1}}(U)=f(u, k) \pi(U)=\mu^{k} \pi(U) \not \varkappa \pi(U)
$$

for all $k$ in $\mathbb{Z}$. Indeed, this holds as the point spectrum of $\pi(U)=D$ is different from the point spectrum of $\pi\left(\alpha_{k^{-1}}(U)\right)=\mu^{k} D$ by construction.

A similar argument also holds if $\nu$ is nontorsion. Hence, we get from Theorem 3.10 that $C^{*}(G, \sigma)$ is primitive if either $\mu$ or $\nu$ is nontorsion.

On the other hand, if $(G, \sigma)$ satisfies condition $\mathrm{K}$, then at least one of $\mu$ and $\nu$ must be nontorsion, so this is also a necessary condition for primitivity of $C^{*}(G, \sigma)$. Indeed, condition (ii) of Proposition 3.1 does not hold if both $\mu$ and $\nu$ are torsion. 
Proposition 3.12. Assume that $\sigma=\sigma_{1} \times \sigma_{2}$ and that both $C^{*}\left(G_{1}, \sigma_{1}\right)$ and $C^{*}\left(G_{2}, \sigma_{2}\right)$ are primitive. Then $C^{*}(G, \sigma)$ is primitive if at least one of $G_{1}$ and $G_{2}$ is amenable.

Proof. Without loss of generality we may assume that $G_{1}$ is amenable. Then $C^{*}\left(G_{1}, \sigma_{1}\right)$ is nuclear by Proposition 2.14 so the minimal and maximal tensor products of $C^{*}\left(G_{1}, \sigma_{1}\right)$ and $C^{*}\left(G_{2}, \sigma_{2}\right)$ coincide. According to [11, Section 3], there is a unique isomorphism

$$
C^{*}(G, \sigma) \rightarrow C^{*}\left(G_{1}, \sigma_{1}\right) \otimes C^{*}\left(G_{2}, \sigma_{2}\right)
$$

given by $i_{G}\left(a_{1}, a_{2}\right) \mapsto i_{G_{1}}\left(a_{1}\right) \otimes i_{G_{2}}\left(a_{2}\right)$.

For $i=1,2$, let $\pi_{i}$ be a faithful irreducible representation of $C^{*}\left(G_{i}, \sigma_{i}\right)$ on $\mathcal{H}_{i}$. Then $\pi=\pi_{1} \otimes \pi_{2}$ is a representation of $C^{*}(G, \sigma)$ on $\mathcal{H}=\mathcal{H}_{1} \otimes \mathcal{H}_{2}$, which is faithful by [17, Theorem 6.5.1] and irreducible by [11, Section 2]. Hence $C^{*}(G, \sigma)$ is primitive.

Remark 3.13. Primitivity of $C^{*}(G, \sigma)$ is in general difficult to decide. For example, let $\mathbb{F}$ be a free nonabelian group, then it is unknown whether $C^{*}(\mathbb{F} \times \mathbb{F})$ is primitive (see [4, Remark 2.2] for a brief discussion).

\section{Free products}

In some sense, free products are easier to treat than direct products, since the Schur multiplier decomposes nicely. Indeed, let $G_{1}$ and $G_{2}$ be two groups. Then we have that (see e.g. [6, page 51])

$$
\mathcal{M}\left(G_{1} * G_{2}\right) \cong \mathcal{M}\left(G_{1}\right) \oplus \mathcal{M}\left(G_{2}\right) .
$$

Let $\sigma_{1}$ be a normalized multiplier on $G_{1}$ and $\sigma_{2}$ a normalized multiplier on $G_{2}$. Following [16, Section 5], we will explain how to obtain a normalized free product multiplier $\sigma_{1} * \sigma_{2}$ on $G_{1} * G_{2}$.

Every nontrivial element $x$ in $G_{1} * G_{2}$ can be uniquely written as a reduced word $x=x_{1} x_{2} \cdots x_{n}$, for which the letters with odd index belong to $G_{i}$ and the letters with even index belong to $G_{j}$ for $i \neq j$. Define the length function as $l(x)=l\left(x_{1} x_{2} \cdots x_{n}\right)=n$ and $l(e)=0$. If $l(x), l(y) \leq 1$, we write $x \perp y$ if either $x=e$ or $y=e$ or else if $x$ is in $G_{i}$ and $y$ is in $G_{j}$ for $i \neq j$.

Let $s(x)$ and $r(x)$ denote the first and last letter of a nontrivial word $x$ and set $s(e)=r(e)=e$. For a pair of words $(x, y)$, we say that the pair is reduced if $r(x) \neq s(y)^{-1}$.

When $(x, y)$ is not reduced, let $w$ be the longest word such that $r\left(x w^{-1}\right) \perp$ $s(w)$ and $r\left(w^{-1}\right) \perp s(w y)$. Set $x_{w}=x w^{-1}$ and $y_{w}=w y$, so that $x=x_{w} w$ and $y=w^{-1} y_{w}$. Let $(x, y)_{w}=\left(x_{w}, y_{w}\right)$ be the reduction of $(x, y)$ and note in particular that $x_{w} y_{w}=x y$.

If the pair $(x, y)$ is reduced, then we set $(x, y)_{w}=(x, y)$.

Define now the multiplier $\tau$ on $G_{1} * G_{2}$ by

$$
\tau(x, y)=\tau\left((x, y)_{w}\right)= \begin{cases}\sigma_{1}\left(r\left(x_{w}\right), s\left(y_{w}\right)\right) & \text { if } r\left(x_{w}\right), s\left(y_{w}\right) \in G_{1} \backslash\{e\}, \\ \sigma_{2}\left(r\left(x_{w}\right), s\left(y_{w}\right)\right) & \text { if } r\left(x_{w}\right), s\left(y_{w}\right) \in G_{2} \backslash\{e\}, \\ 1 & \text { if } r\left(x_{w}\right) \perp s\left(y_{w}\right),\end{cases}
$$


and note that this definition coincides with the one explained in [16. Section 5]. Furthermore, let

$$
X=\left\{[a, b]=a b a^{-1} b^{-1}: a \in G_{1} \backslash\{e\}, b \in G_{2} \backslash\{e\}\right\}
$$

and recall that the free nonabelian group on $X$, denoted $\mathbb{F}_{X}$, may be identified with the normal subgroup of $G_{1} * G_{2}$ generated by $X$.

Moreover, define a function $\beta: G_{1} * G_{2} \rightarrow \mathbb{T}$ by $\beta(x)=1$ if $x \notin \mathbb{F}_{X}$, while for nontrivial $x=q_{1}^{p_{1}} \cdots q_{n}^{p_{n}}$ in $\mathbb{F}_{X}$, where $q_{i}$ belongs to $X$ and $p_{i}$ is an integer, we set

$$
\beta(x)=\beta\left(q_{1}^{p_{1}} \cdots q_{n}^{p_{n}}\right)= \begin{cases}\tau\left(q_{1}^{p_{1}}, q_{2}^{p_{2}}\right) \tau\left(q_{2}^{p_{2}}, q_{3}^{p_{3}}\right) \cdots \tau\left(q_{n-1}^{p_{n-1}}, q_{n}^{p_{n}}\right) & \text { if } n \geq 2, \\ 1 & \text { if } n=1 .\end{cases}
$$

Now define the multiplier $\sigma$ on $G_{1} * G_{2}$ by

$$
\sigma(x, y)=\beta(x) \beta(y) \overline{\beta(x y)} \tau(x, y) .
$$

We write $\sigma=\sigma_{1} * \sigma_{2}$ and note that $\sigma \sim \tau, \sigma_{\mid G_{i} \times G_{i}}=\sigma_{i}$ and $\sigma_{\mid \mathbb{F}_{X} \times \mathbb{F}_{X}}=1$.

On the other hand, if $\sigma$ is a normalized multiplier on $G_{1} * G_{2}$, we can define the restriction $\sigma_{1}$ on $G_{1}$ by

$$
\sigma_{1}(x, y)= \begin{cases}\sigma(x, y) & \text { if } x, y \in G_{1} \backslash\{e\} \\ 1 & \text { if } x \text { or } y=e\end{cases}
$$

Similarly, we can define the restriction $\sigma_{2}$ of $\sigma$ to $G_{2}$. Next, define the function $\beta: G_{1} * G_{2} \rightarrow \mathbb{T}$ by $\beta(x)=1$ if $l(x) \leq 1$ and else

$$
\beta(x)=\beta\left(x_{1} \cdots x_{n}\right)=\sigma\left(x_{1}, x_{2}\right) \sigma\left(x_{1} x_{2}, x_{3}\right) \cdots \sigma\left(x_{1} \cdots x_{n-1}, x_{n}\right) .
$$

Then $\sigma$ is similar to $\sigma_{1} * \sigma_{2}$ through $\beta$.

Remark that every multiplier is similar to a normalized one. Therefore, every multiplier on $G_{1} * G_{2}$ is similar to $\sigma_{1} * \sigma_{2}$ for some normalized multipliers $\sigma_{1}$ on $G_{1}$ and $\sigma_{2}$ on $G_{2}$.

We are now ready to prove the twisted version of [3, Theorem 1.2].

Theorem 4.1. Assume $G=G_{1} * G_{2}$, where $G_{1}$ and $G_{2}$ are countable and amenable and $\left(\left|G_{1}\right|-1\right)\left(\left|G_{2}\right|-1\right) \geq 2$, and let $\sigma$ be a multiplier on $G$. Then $C^{*}(G, \sigma)$ is primitive.

Proof. We may assume that $\sigma=\sigma_{1} * \sigma_{2}$ where $\sigma_{1}$ and $\sigma_{2}$ are normalized multipliers on $G_{1}$ and $G_{2}$, respectively, and that $\sigma_{\mid \mathbb{F}_{X} \times \mathbb{F}_{X}}=1$. The proof is only a slight modification of the proof of [3. Theorem 1.2], so we just point out what needs to be adjusted in this proof and use the notation therein. First, recall that there is a twisted action $(\alpha, \omega)$ of $\left(G_{1} * G_{2}\right) / \mathbb{F}_{X} \cong G_{1} \times G_{2}$ on $H=\mathbb{F}_{X}$. Straightforward calculations give that

$$
\alpha_{(c, d)}\left(i_{H}([a, b])\right)= \begin{cases}i_{H}\left(c d[a, b] d^{-1} c^{-1}\right) \cdot \sigma_{2}(d, b) & \text { if } d \neq e \\ i_{H}\left(c d[a, b] d^{-1} c^{-1}\right) \cdot \sigma_{1}(c, a) & \text { if } d=e\end{cases}
$$

for $a, c \in G_{1}$ and $b, d \in G_{2}$. Hence the expressions in the equations [3, (2.3),(2.4)] remain unchanged, so it is enough to reconsider 3. Case 3]. More straightforward calculations give that the conditions at the bottom of [3, page 54] must be 
replaced with:

$$
\begin{aligned}
& k=\left(s_{0}, t\right) \text { and } k=\left(s_{l}, e_{2}\right) \text { if } \\
& \lambda\left(s_{0} s_{l}, t\right) U\left(s_{0} s_{l}, t\right) \not \sigma_{1}\left(s_{l}, s_{0} s_{l}\right) U\left(s_{0}, t\right)\left(\lambda\left(s_{l}, t\right) U\left(s_{l}, t\right)\right)^{*} ; \\
& k=\left(s_{0}, e_{2}\right) \text { and } k=\left(s_{l}, t\right) \text { if } \\
& \lambda\left(s_{0} s_{l}, t\right) U\left(s_{0} s_{l}, t\right) \not \sigma_{1}\left(s_{0}, s_{0} s_{l}\right) \lambda\left(s_{l}, t\right) U\left(s_{l}, t\right) U\left(s_{0}, t\right)^{*} ; \\
& k=\left(s_{0}, t\right) \text { and } k=\left(s_{0} s_{l}, e_{2}\right) \text { if } \\
& \lambda\left(s_{l}, t\right) U\left(s_{l}, t\right) \neq \sigma_{1}\left(s_{0} s_{l}, s_{l}\right) U\left(s_{0}, t\right)\left(\lambda\left(s_{0} s_{l}, t\right) U\left(s_{0} s_{l}, t\right)\right)^{*} ; \\
& k=\left(s_{0} s_{l}, t\right) \text { and } k=\left(s_{0}, e_{2}\right) \text { if } \\
& \lambda\left(s_{l}, t\right) U\left(s_{l}, t\right) \not \sigma_{1}\left(s_{0}, s_{l}\right) \lambda\left(s_{0} s_{l}, t\right) U\left(s_{0} s_{l}, t\right) U\left(s_{0}, t\right)^{*} .
\end{aligned}
$$

Now it is easily seen that the rest of the proof works with appropriate modifications.

Remark 4.2. Theorem 4.1 is not surprising. In fact, I am not aware of any pair $(G, \sigma)$ such that $C^{*}(G)$ is primitive, but $C^{*}(G, \sigma)$ is nonprimitive.

Remark 4.3. Let $G=G_{1} * G_{2}$, let $\sigma$ be a multiplier on $G$ and assume $\sigma=\sigma_{1} * \sigma_{2}$. Then it is known that (see [16, Section 5])

$$
C^{*}(G, \sigma)=C^{*}\left(G_{1}, \sigma_{1}\right) * C^{*}\left(G_{2}, \sigma_{2}\right) .
$$

Example 4.4. As explained in Example 1.1 we have that for each natural number $n$, there exists a multiplier $\sigma_{k}$ on $\mathbb{Z}_{n} \times \mathbb{Z}_{n}$ such that $C^{*}\left(\mathbb{Z}_{n} \times \mathbb{Z}_{n}, \sigma_{k}\right) \cong$ $M_{n}(\mathbb{C})$. One immediate consequence of Theorem 4.1 is that

$$
M_{j}(\mathbb{C}) * M_{k}(\mathbb{C})
$$

is primitive for all $j, k \geq 2$. More generally, it has recently been shown 10 that $F_{1} * F_{2}$ is primitive whenever $F_{1}$ and $F_{2}$ are finite-dimensional $C^{*}$-algebras and $\left(\operatorname{dim} F_{1}-1\right)\left(\operatorname{dim} F_{2}-1\right) \geq 2$.

\section{References}

[1] Erik Bédos. Notes on hypertraces and $C^{*}$-algebras. J. Operator Theory, 34(2):285-306, 1995.

[2] Erik Bédos and Roberto Conti. On twisted Fourier analysis and convergence of Fourier series on discrete groups. J. Fourier Anal. Appl., 15(3):336$365,2009$.

[3] Erik Bédos and Tron Å. Omland. Primitivity of some full group $C^{*}$-algebras. Banach J. Math. Anal., 5(2):44-58, 2011.

[4] Erik Bédos and Tron $\AA$. Omland. The full group $C^{*}$-algebra of the modular group is primitive. Proc. Amer. Math. Soc., 140(4):1402-1411, 2012.

[5] M. Bekka, M. Cowling, and P. de la Harpe. Simplicity of the reduced $C^{*}$ algebra of $\operatorname{PSL}(n, \mathbb{Z})$. Internat. Math. Res. Notices, (7):285ff., approx. 7 pp. (electronic), 1994. 
[6] Kenneth S. Brown. Cohomology of groups, volume 87 of Graduate Texts in Mathematics. Springer-Verlag, New York, 1982.

[7] Man Duen Choi. The full $C^{*}$-algebra of the free group on two generators. Pacific J. Math., 87(1):41-48, 1980.

[8] Alain Connes. Classification of injective factors. Cases $\mathrm{II}_{1}, \mathrm{II}_{\infty}, \mathrm{III}_{\lambda}, \lambda \neq 1$. Ann. of Math. (2), 104(1):73-115, 1976.

[9] Jacques Dixmier. Sur les $C^{*}$-algèbres. Bull. Soc. Math. France, 88:95-112, 1960.

[10] Ken Dykema and Francisco Torres-Ayala. Primitivity of unital full free products of finite dimensional $C^{*}$-algebras. preprint, 2012.

[11] Alain Guichardet. Tensor products of $C^{*}$-algebras: Finite tensor products, volume 12 of Lecture notes series. Aarhus Universitet, Matematisk Institut, 1969.

[12] Richard V. Kadison and John R. Ringrose. Fundamentals of the theory of operator algebras. Vol. II, volume 100 of Pure and Applied Mathematics. Academic Press Inc., Orlando, FL, 1986. Advanced theory.

[13] Adam Kleppner. The structure of some induced representations. Duke Math. J., 29:555-572, 1962.

[14] V. Losert. On the center of group $C^{*}$-algebras. J. Reine Angew. Math., 554:105-138, 2003.

[15] George W. Mackey. Unitary representations of group extensions. I. Acta Math., 99:265-311, 1958.

[16] Kevin McClanahan. $K K$-groups of twisted crossed products by groups acting on trees. Pacific J. Math., 174(2):471-495, 1996.

[17] Gerard J. Murphy. $C^{*}$-algebras and operator theory. Academic Press Inc., Boston, MA, 1990.

[18] Gerard J. Murphy. Primitivity conditions for full group $C^{*}$-algebras. Bull. London Math. Soc., 35(5):697-705, 2003.

[19] Francis Joseph Murray and John von Neumann. On rings of operators. IV. Ann. of Math. (2), 44:716-808, 1943.

[20] Judith A. Packer. $C^{*}$-algebras generated by projective representations of the discrete Heisenberg group. J. Operator Theory, 18(1):41-66, 1987.

[21] Judith A. Packer. Twisted group $C^{*}$-algebras corresponding to nilpotent discrete groups. Math. Scand., 64(1):109-122, 1989.

[22] Judith A. Packer and Iain Raeburn. Twisted crossed products of $C^{*}$ algebras. Math. Proc. Cambridge Philos. Soc., 106(2):293-311, 1989.

[23] Joseph Slawny. On factor representations and the $C^{*}$-algebra of canonical commutation relations. Comm. Math. Phys., 24:151-170, 1972. 
[24] Georges Zeller-Meier. Produits croisés d'une $C^{*}$-algèbre par un groupe d'automorphismes. J. Math. Pures Appl. (9), 47:101-239, 1968.

Department of Mathematical Sciences, Norwegian University of Science and Technology (NTNU), NO-7491 Trondheim, Norway.

E-mail address: tron.omland@math.ntnu.no 